\title{
Lung carcinoid tumor biology: Treatment and survival
}

\author{
J. DAHABREH ${ }^{1}$, G.P. STATHOPOULOS ${ }^{2}$, J. KOUTANTOS ${ }^{2}$ and S. RIGATOS ${ }^{1}$ \\ ${ }^{1}$ Thoracic Surgery Clinic, 'Iatriko Center', Athens; ${ }^{2}$ First Oncology Clinic, Errikos Dunant Hospital, Athens, Greece
}

Received September 30, 2008; Accepted December 22, 2008

DOI: 10.3892/or_00000281

\begin{abstract}
A carcinoid tumor is a rare malignant disease which can be cured when localized and treated by surgery. Chemotherapy is not effective, and somatostatin is used for palliation. Rarely is the disease aggressive, and thus does not contribute to a shortening of patient survival. The aim of this study was to define the treatment and survival of patients with primary lung carcinoid tumors. Forty-three patients (26 males, 17 females; median age 43 years, range 11-73 years), from 1993 to 2007, were included in this study. All patients had histologically confirmed carcinoid tumors. The site of the disease at diagnosis was the lung in all 43 patients. All patients underwent surgery which involved mainly typical or sleeve lobectomy. Eight patients had a pneumonectomy. One patient had the primary tumor excised for palliation as there were metastases in the liver. Somatostatin palliative treatment was administered to 4 patients; 1 with liver and 3 with lung recurrence. Two of the 43 patients died within 2 years after surgery. The median survival was not reached as all patients, apart from 2, were alive after a median follow-up of 5 years (mean survival 159 months). As a rule, a carcinoid tumor is an extremely slow-growing disease with some rare exceptions. All of our patients had primary lung disease. All, apart from 2 , were alive at the end of the study, and $93 \%$ were without recurrence for a duration of 6 months to 13 years. The patients with liver metastases who underwent no specific treatment had a median survival as long as 8 years.
\end{abstract}

\section{Introduction}

A carcinoid tumor is a rare malignant disease which is often cured by surgery when it is localized. Carcinoid tumors represent $2 \%$ of all pulmonary neoplasms (1). The majority of these tumors (90\%) are confined to the bronchus and $10 \%$ are located in the lung parenchyma, while rarely being located in the main carina or in the trachea.

Carcinoid tumors are classified as neuroendocrine tumors, and the vast majority are characterized as typical carcinoids,

Correspondence to: Dr G.P. Stathopoulos, Semitelou 2A, 11528 Athens, Greece

E-mail: dr-gps@ath.forthnet.gr

Key words: lung, carcinoid tumor while the remainder are atypical carcinoids (2). Carcinoids, as other neuroendocrine tumors, may secrete hormone-like substances such as adenocorticotropic hormone (ACTH) and arginine vasopressin, and in these cases paraneoplastic syndromes accompany the disease (3). Rarely is the disease aggressive, and thus does not contribute to a shortening of patient survival. Aggressive cases are mainly classified as an atypical type of carcinoid which is likely to metastasize to the regional lymph nodes and/or to the liver, bone and brain (4). The metastatic tendency of the typical carcinoid is approximately $5 \%$.

In past years, these tumors were considered to be benign, similar to adenomas, until metastases were observed (5). According to the latest WHO classification, they are classified as malignant tumors. Despite the fact that these tumors are malignant, their behavior is usually benign and are rarely responsible for the death of patients even after an extensive period of follow-up.

This disease may involve children and adults, males and females (ratio 1.6:1), and the treatment of choice is surgery for localized disease. In advanced or metastatic disease no effective treatment exists. Chemotherapy with a cisplatinbased or streptozotocin-based combination is often administered. Biotherapy including interferon, somatostatin analogs and octreotide is used in carcinoid management (6) for palliation or the relief of symptoms. Octreotide, a somatostatin analog, may relieve pain (7), but a positive octreoscanning is required before treatment.

Neuroendocrine tumors of the lung are classified into well differentiated, typical and atypical carcinoids and a poorly differentiated subgroup which includes large-cell neuroendocrine carcinomas and small-cell carcinomas; the latter of which are well known for their aggressive behavior (8). According to the WHO classification (2), typical and atypical carcinoids differ in the number of mitoses $(<2$ per 10 HPF vs. 2-10 per $10 \mathrm{HPF}$ ), in nuclear polymorphism which is either absent or present, in the percentage of lymph node metastasis which is either low or high, and in the occurrence of either rare or common metastatic disease, at presentation, respectively.

The aim of this study was to determine tumor evolution and patient survival.

\section{Materials and methods}

From 1993 to 2007, 43 patients were admitted to the Thoracic Surgery Department, Iatriko Center, Athens for diagnosis and treatment of lung disease. Some of the patients presented facial flushing and occasional diarrhea, but most were 
Table I. Patient characteristics.

\begin{tabular}{lc}
\hline Characteristic & No. of patients $(\mathrm{n}=43)$ \\
\hline Age (years) & 43 \\
Median & $11-73$ \\
Range & \\
Gender & 26 \\
Male & 17 \\
Female & \\
Histology & 36 mitoses $\left(\leq 2 / 2\right.$ mm $\left.^{2}\right)$ \\
Typical carcinoid & 7 mitoses $(>2$ to $>10)$ \\
Atypical carcinoid & \\
Disease stage & 25 localized \\
I + II & 11 lymph node location \\
IIIa & 6 lymph node + pleura \\
IIIb & 1 liver metastasis \\
IV &
\end{tabular}

symptomless. In all cases, a chest X-ray and computed tomography of the chest and upper abdomen were performed, as well as a bronchoscopy. Full blood count and liver and renal function tests were within normal values. ECG and respiratory function tests were also performed. The lung tumor was detected, and the diagnosis was confirmed histologically or cytologically in $90 \%$ of the patients before surgery. For the diagnosis, a biopsy using a flexible bronchoscope was performed. All patients were considered operable. The patient characteristics are shown in Table I. Urinary 5-hydroxyindoloacetic acid values were determined after diagnosis, and only one patient with liver metastatic disease had an increased value.

Treatment. All 43 patients were treated by means of radical surgical resection, to different extents, depending on the location of the tumor, the patient's age, the parenchymal condition of the lung and the pulmonary function results. Table II lists the types of surgical intervention. Our intent was to perform curative surgery. One patient had liver metastasis, with an extensively damaged right lung because of the central location of the tumor, and this patient had recurrent infections; the operation in this case was performed mainly for palliative reasons. The most frequently performed operation was typical lobectomy followed by sleeve lobectomy. Sleeve resection was performed in every case when the tumor was located at the orifice of a lobar or a main bronchus. Pneumonectomy was performed in every case when the tumor was located at the main bronchus with extensive damage to the lung due to prolonged atelectasis and recurrent infections, as well as in every case of infiltrated and fixed interlobar lymph nodes. Sleeve lobectomy or resection of the bronchus without the resection of lung parenchyma was performed whenever the radical resection of the tumor was feasible, even when the patients had sufficient pulmonary reserves to allow a pneumonectomy. Pleuropneumonectomy
Table II. Surgical treatment.

\begin{tabular}{lc}
\hline Type of surgical intervention & $\begin{array}{c}\text { No. of patients } \\
(\mathrm{n}=43)\end{array}$ \\
\hline Wedge resection & 1 \\
Typical lobectomy & 14 \\
Sleeve lobectomy & 8 \\
Typical middle and lower bilobectomy & 4 \\
Sleeve bilobectomy (middle and lower) & 1 \\
Tracheal resection & 1 \\
Typical pneumonectomy & 8 \\
Sleeve pneumonectomy & 1 \\
Pleuropneumonectomy & 3 \\
Bronchial resection & \\
(without parenchymal resection) & 2 \\
\hline
\end{tabular}

was performed in three cases upon recurrence of the tumors; all 3 patients were female and had undergone initial surgical procedure 10 to 16 years prior to the recurrence. Two of these patients underwent middle lobectomy while the third had a segmentectomy of the middle lobe. All three cases had the same pattern of recurrence which was at the intermedius bronchus with multiple deposits throughout the pleural cavity including the parietal and visceral pleura. In two of the three cases a pleuropneumonectomy was performed in combination with the resection of the diaphragm and the pericardium which were reconstructed by the insertion of a synthetic graft. The third patient underwent a pleuropneumonectomy without the need of a pericardiectomy or diaphragm resection, because of the limited dissemination throughout the pleural cavity.

Wedge resection was performed in one case where the tumor was $<1 \mathrm{~cm}$ in diameter and was discovered during a right thoracotomy conducted as treatment for non-small cell lung cancer, and the carcinoid tumor was palpated and resected as an incidental intraoperative finding. Tracheal resection was performed for a carcinoid tumor of the trachea located $3 \mathrm{~cm}$ below the vocal cords, and an end-to-end anastomosis was performed after the resection of a $4-\mathrm{cm}$ length of trachea. We performed a right sleeve pneumonectomy because of the infiltration of the right main bronchus and the carina.

There were no perioperative deaths. Postoperative complications (Table III) were observed in 7 of 43 (16.28\%) patients all of whom were treated effectively.

Follow-up. The median follow-up was 54 months (range 6-168 months). At the end of the study analysis there were only two deaths observed due to advanced disease. Two of the patients had liver metastasis confirmed by biopsy, and one had recurrence at the anastomosis site. The recurrence of disease was diagnosed at 5 and 6 years, respectively, after the lung operation (lobectomy and sleeve pneumonectomy). These patients were treated with somatostatin. The other 39 patients remain free of disease. 
Table III. Postoperative complications.

\begin{tabular}{lc}
\hline Complication & No. of patients \\
\hline Rupture of anastomosis & 1 \\
Atrial fibrillation & 1 \\
Respiratory infection & 1 \\
Pericardial effusion & 1 \\
Asthmatic crisis & 1 \\
Hemorrhage & 1 \\
Atelectasis & 1 \\
Total & 7 \\
\hline
\end{tabular}

\section{Results}

Survival. Long-term survival was achieved in patients with localized typical or atypical carcinoid tumors. The main difference between the two types was that the atypical was locally more advanced and led to more extensive surgery, i.e. pneumonectomy (lymph nodes involved), instead of a lobectomy. Of the 3 patients with liver metastasis, the first had a rather short survival of 2 years while the other two were still alive at the end of the study (9 years and 8 months, respectively), following detection of metastatic disease in the liver. Somatostatin treatment did not seem to contribute to the patient survival. When the present study was integrated, 41 out of 43 patients were alive and healthy. Two of the 41 patients had disease recurrence. Fifty percent of the patients were disease-free for $>54$ months. The mean survival was 159 months (range 6-168 months). Fig. 1 shows the KaplanMeier survival curve.

\section{Discussion}

Primary carcinoid tumor of the lung is a rare disease. Surgical resection remains the cornerstone in the treatment of carcinoids of the lung. We performed a broad spectrum of surgical procedures in order to achieve two aims: firstly, radical resection and secondly the least resection possible of the normally functioning lung parenchyma. We performed pleuropneumonectomy in three cases because of local recurrence in the intermedius bronchus after a prior lobectomy in 2 patients and segmentectomy in a third patient. All three of these patients had undergone surgery for a typical carcinoid of the middle bronchus 10-16 years before recurrence. These 3 patients had the same pattern of recurrence: intrabronchial recurrence and dissemination throughout the pleural cavity, with multiple deposits of the parietal and visceral pleura. Pleuropneumonectomy as a treatment for lung carcinoid tumors has not been previously described in the medical literature. One of the three above-mentioned patients who underwent pleuropneumonectomy, a 22-year-old female, has been free of disease for 6 years following the surgical procedure and the other 2 , also young female patients, have been free of disease for 6 and 8 months, respectively, following pleuropneumonectomy.

In regards to carcinoid tumors of the lung, there have been a few studies with a small number of patients. Long-term survival has been reported in studies where the tumor was resected. The difference in survival for patients with typical and atypical carcinoid tumors has been documented. In one study, the 3- and 5-year survival was 100 and $81 \%$, respectively, for typical tumors, and 96 and $68 \%$, respectively, for atypical tumors (8). Similar findings were also found in another study focusing on the survival of patients with liver metastases; patient survival varied from 16 to 51 months (4). An extensive

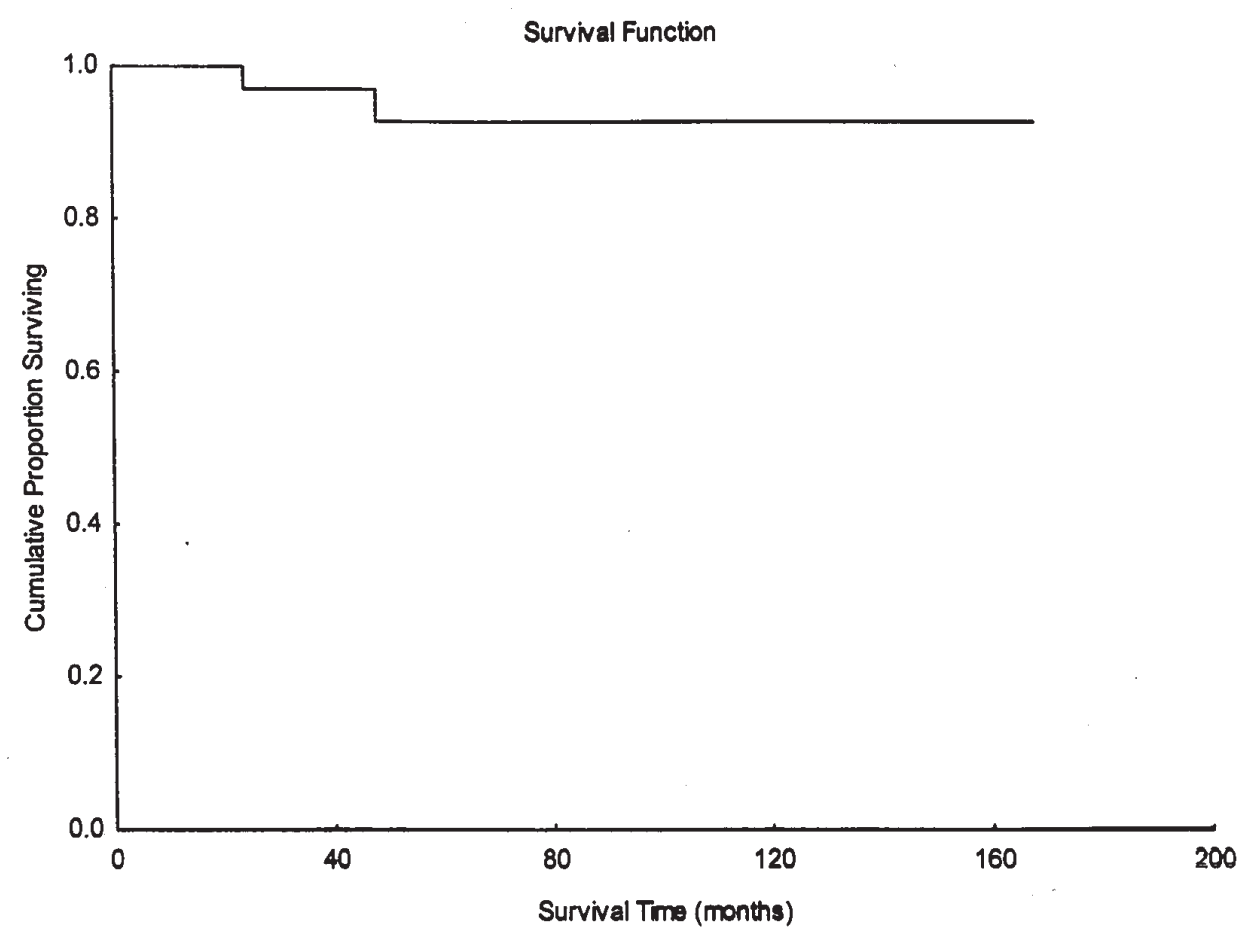

Figure 1. Kaplan-Meier survival curve. 
review and analysis of patient characteristics and survival found that atypical carcinoids occur in no more than $15 \%$ of cases (9). There is a large age distribution, and the disease involves males more often than females. These researchers reported a 93.3\% 5-year survival rate and an $82.1 \%$ 10-year survival. In our study, long-term survival was also observed; a 41.9\% 5-year survival and an 18.6\% 10-year survival [bearing in mind that 41 out of $43(95.35 \%)$ patients were alive and healthy when the study was integrated]. The present data justify the past consideration as to whether or not typical carcinoid syndrome is a malignant disease. All patients who presented with metastases in our study, as well as in other studies, were classified as having atypical carcinoids which could be a different disease on the basis of biological behavior.

In conclusion, we contributed to a greater understanding of carcinoid tumor biology and its therapeutic management by describing an adequate number of cases with this rare tumor.

\section{References}

1. Chugta T, Morin J, Sheiner N, Wilson J and Mulder D: Bronchial carcinoid: twenty years experience defines selective surgical approach. Surgery 122: 801-808, 1997.

2. Travis WD, Corrin B, Shimosato Y and Brambilla E: The histological typing of lung and pleural tumors. In: WHO/IASLC Classification of Lung and Pleural Tumors. 3rd edition, Springer, Berlin, 1999.

3. Yellin A and Benfield JR: The pulmonary Kultchisky cell (neuroendocrine) cancers: from carcinoid to small cell carcinoma. Curr Probl Cancer 9: 1-38, 1985.

4. Filosso PL, Ruffini E, Oliaro A, Papalia E, Donati G and Rena O: Long-term survival of atypical bronchial carcinoids with liver metastases, treated with octreotide. Eur J Cardiothorac Surg 21: 913-917, 2002.

5. Arrigoni MG, Woolner LB and Bernatz PE: Atypical carcinoid tumors of the lung. J Thorac Surg 3: 413-421, 1972.

6. Kosmidis PA: Treatment of carcinoid of the lung. Curr Opin Oncol 16: 146-149, 2004.

7. Katai M, Sakurai A, Inaba H, Ikeo Y, Yamauchi K and Hashizume K: Octreotide as a rapid and effective painkiller for metastatic carcinoid tumor. Endocr J 52: 277-280, 2005.

8. Divisi D and Crisci R: Carcinoid tumors of the lung and multimodal therapy. Thorac Cardiovasc Surg 53: 168-172, 2005.

9. Soga $J$ and Yakuwa Y: Bronchopulmonary carcinoids: an analysis of 1875 reported cases with special reference to a comparison between typical carcinoids and atypical varieties. Ann Thorac Cardiovasc Surg 5: 211-219, 1999. 